\title{
O CUSTO DA DOENÇA: REPERCUSSÃO ECONÔMICA NO MUNICÍPIO DE SALVADOR, BA, BRASIL *
}

José Duarte de Araújo **

RSPU-B $/ 256$

\begin{abstract}
DUARTE DE ARAÚJo, J. - o custo da doença: repercussão económica no municipio de Salvador, BA, Brasil. Rev. Saúde públ., S. Paulo, 9:155-68, 1975.
\end{abstract}

RESUMO: Estudou-se as repercussōes econômicas da mortalidade no municipio de Salvador, Brasil, no ano de 1970. Procurou-se comparar a mortalidade de Salvador com a de Porto Alegre e avaliar o custo do excesso de mortalidade em Salvador. Os resultados das estimativas mostraram ser acentuado o prejuizo econômico resultante da mortalidade por doenças passiveis de prevenção, levando à conclusão de que os gastos com o setor saúde devem ser vistos como investimento e não como despesa de consumo.

Unitermos: Custo da doença. Economia médica. Salvador, Brasil.

\section{I N T R O D U C A O}

Com o objetivo de avaliar o custo da doença, dada a dificuldade de obter dados em escala nacional, foi estudado um aspecto do problema, a mortalidade, utilizando dados do município do Salvador. BA. Brasil. Não foi abordado o custo da morbidade. a exemplo de Rice ${ }^{15}$, pela ausência dos dados estatísticos indispensáveis.

\section{MATERIAL E METODOS}

\subsection{Fonte dos Dados}

As informaçôes referentes aos óbitos por causa. sexo e grupos etários, em Salvador. no ano de 1970. foram obtidos no Serviço de Bioestatística da Secretaria de Saúde Pública da Bahia. Para efeito de comparação. os dados de mortalidade em
Porto Alegre, em 1970, foram retirados do Anuário Estatístico do IBGE ${ }^{4}$, em virtude de não ter sido possível obtê-los diretamente $\mathrm{da}$ fonte original, ou seja, do Serviço de Bioestatística da respectiva Secretaria de Saúde.

As informações referentes à participação na força de trabalho e níveis de salário foram obtidas dos dados publicados pela Pesquisa Nacional por Amostra de Domicílios" Os dados de esperança de vida para a população de Salvador foram colhidos da pesquisa feita pelo Programa de Recursos Humanos da Inniversidade Federal da Baria ${ }^{16}$.

\subsection{Método de Estimativa}

Dada a falta de informações sobre os perfís de renda por grupos etários. foi-nos

* Parte da Tese intitulada Aspectos Económicos da Saúde apresentada à Universidade da Bahia para concurso de Professor Titular, em junho de 1974

** Chefe do Departamento de Medicina Preventiva da Faculdade de Medicina da Universidade Federal da Bahia - Parque Universitário, Canela. Salvador, BA - Brasil 
DUARTE DE ARAGJO, J. - o custo da doença: repercussão econômica no município de Salvador, BA, Brasil, Rev. Saúde públ., S. Paulo, 9:155-68, 1975.

impossível aplicar no cálculo dos prejuizos econômicos decorrentes da mortalidade, a fórmula preconizada por Rice ${ }^{15}$, ou valer-nos do método de Johnson ${ }^{8}$, que, em 1970 desenvolveu um modelo para predizer os ganhos futuros de um individuo com base em perfís de salário por idade e nível educacional, o qual também dificilmente poderia ser aplicado no Brasil por falta de dados estatísticos indispensáveis. Ao invés disto, tivemos que desenvolver uma metodologia simples de cálculo, utilizando apenas o número de óbitos em cada grupo etário, a vida média esperada naquela idade, a participação da força de trabalho do grupo etário de 14 a 64 anos e o valor médio dos salários destes indivíduos, separadamente, para ambos os sexos. O número de anos de vida produtiva perdidos foi calculado multiplicando-se o número de óbitos em cada grupo etário pela diferença entre a vida média esperada naquela faixa etária e a idade central do referido grupo, descontando os anos abaixo de 14 e acima de 64 , geralmente aceitos pelos economistas como período não produtivo (Tabela 1 ). Em se tratando de dados referentes ao município de Salvador, zona quase que exclusivamente urbana, não se aplica aqui a objeção da entrada na força de trabalho com menos de 14 anos que é freqüente em zonas rurais. 0 somatório dos anos de vida potencialmente produtiva foi então multiplicado pelo valor percentual de ocupação da força de trabalho, obtendo-se assim o número de anos efetivos de trabalho produtivo perdidos em conseqüência da mortalidade precoce. Este cálculo foi realizado separadamente para homens e mulheres, sendo que no sexo feminino foram consideradas duas hipóteses:

a) não incluindo as mulheres ocupadas em afzzeres domésticos (donas de casa) ;

b) incluindo as atividades domésticas como participação efetiva na força de trabalho.
Os percentuais de ocupação foram: $88,17 \%$ para o sexo masculino, $38,02 \%$ para o sexo feminino sem incluir as atividades domésticas, e $90,34 \%$ para o sexo feminino incluindo as atividades domésticas.

O valor monetário do total de anos produtivos perdidos para a economia foi calculado multiplicando-se o número de anos perdidos para cada sexo, pelo salário anual médio em 1970 estimado a partir de dados da Pesquisa Nacional por Amostra de Domicílios $^{5}$, o qual foi de Cr\$2.681,12 para o sexo masculino e de Cr $\$ 1.610,96$ para o sexo feminino.

A metodologia acima exposta representa uma tomada de posição definida quanto a duas questões abordadas na revisão de literatura: o problema do desemprego e o da taxa de desconto (Duarte de Araújo ${ }^{2}$ ).

No que diz respeito ao problema do desemprego que, segundo alguns economistas, invalidaria a tentativa de atribuir uma perda econômica à morte de indivíduos na faixa de idade potencialmente produtiva, em países economicamente subdesenvolvidos, tomamos a posição de não aceitar esta premissa. Em primeiro lugar, julgamos que os dados da literatura revista dão suficiente justificativa ao ponto de vista de que a produtividade marginal do trabalho no nosso tipo de economia é positiva $^{6,11,13}$. Em segundo lugar, concordamos com aqueles que julgam que é responsabilidade do setor saúde cuidar de manter a higidez e o bem estar da força de trabalho, independente de qual seja a sua eventual utilização nos setores produtivos da economia ${ }^{3}$. Finalmente, tivemos o cuidado de levar em conta, em nosso cálculo, as taxas de participação efetiva da força de trabalho nas atividades produtivas ou seja, ao invés de admitir que todo indivíduo que não tivesse morrido precocemente estaria empregado, produzindo, admitimos que aqueles indivíduos, se vivos e hígidos, teriam a mesma oportunidade de trabalhar e produzir que o restante da população. 
DUARTE DE ARAOUJO, J. - O custo da doença: repercussão económica no municipio de Salvador, BA, Brasil. Rev. Saúde puibl., S Paulo, 9:155-68, 1975

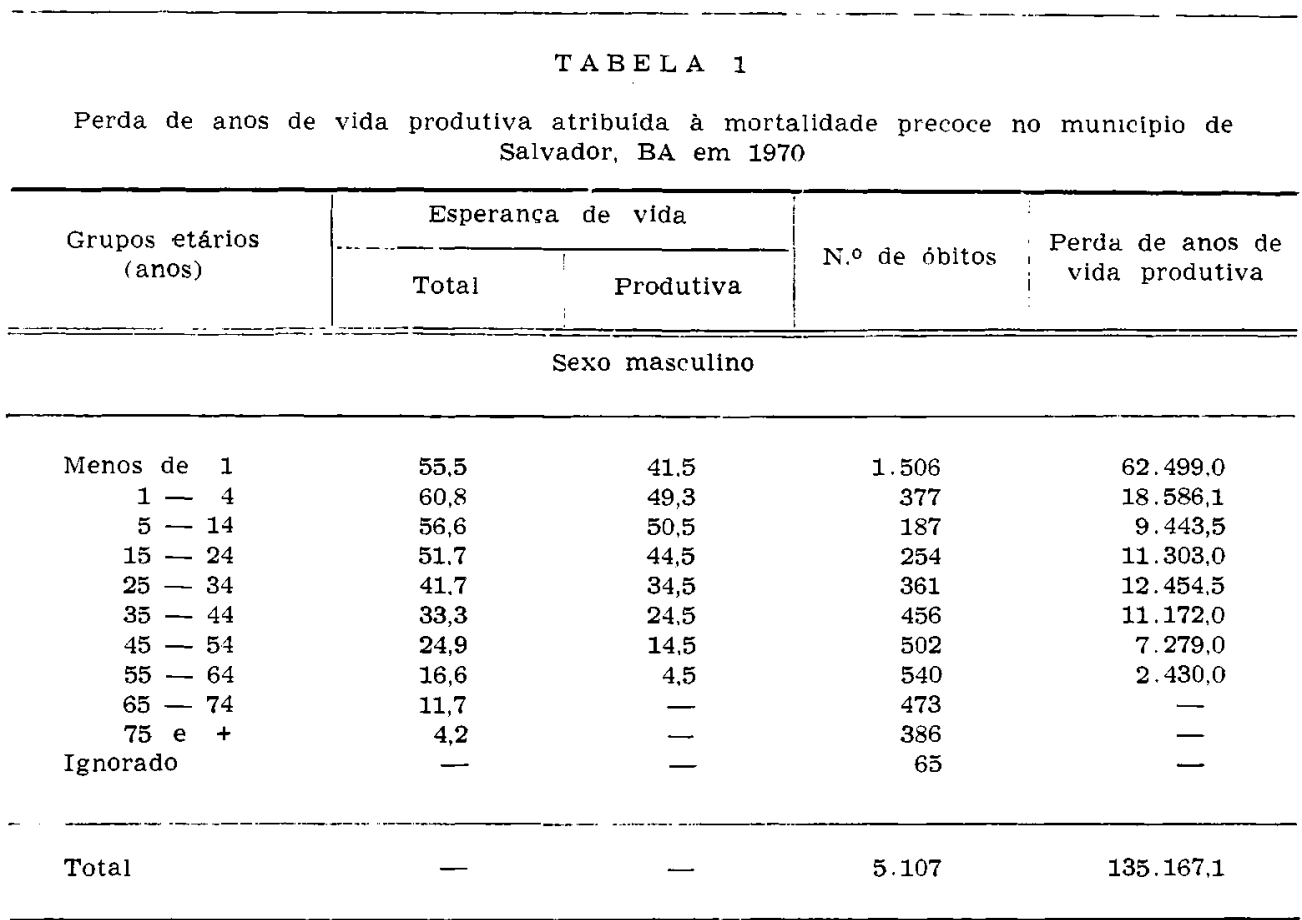

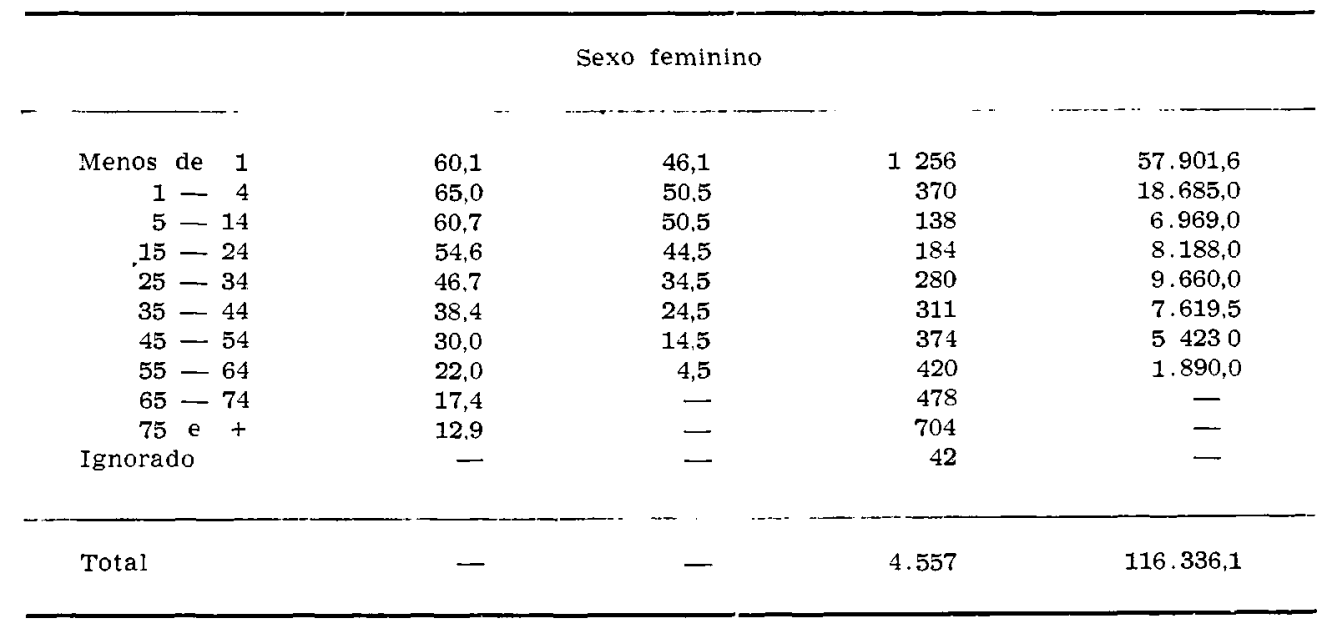

De referência à questão do uso de uma taxa de desconto para o cálculo do valor presente dos ganhos futuros, a situação mostra-se complicada em nosso pais pela taxa de inflação. Se, de acordo com a recomendação de Klarman ${ }^{10}$, fôssemos abater da taxa de desconto a taxa de inflação, teríamos que aplicar no final não uma taxa de desconto mas sim uma correção monetária, pois no momento a taxa de inflação é ainda superior às taxas de desconto comumente utilizadas neste tipo de estudo. Ou seja, com uma taxa anual de inflação de $19.8 \%$, válida para o Brasil em 1970, caso resolvêssemos usar uma taxa de descontos de $10 \%$, teríamos na realidade de aplicar uma taxa de juros de $8,2 \%$ ao ano aos valores dos ganhos futuros para obtermos o seu valor presente. Esta hipótese. embora teoricamente 
DUARTE DE ARAOJO, J. - O custo da doenca: repercussão econômica no municipio de Salvador, BA, Brasll. Rev. Saúde públ., S. Paulo, 9:155-68, 1975.

razoável, parece-nos não justificada na prática pela situação de nossa economia em que se verificam modificações constantes das taxas tanto de desconto quanto de inflação, sendo a rigor imprevisível o comportamento das referidas taxas após duas ou três décadas. Ademais, Miller ${ }^{12}$, estudando dados longitudinais de renda pessoal em diferentes grupos etários entre 1950 e 1960 , considerou que o cálculo dos ganhos futuros, com base em dados de cortes transversais de população, subestima o seu valor por não levar em conta o efeito do progresso e da educação sobre os salários individuais, particularmente nos grupos etários mais jovens. Segundo ele esta tendência a subestimar é agravada pelo uso de taxas de desconto. Consideramos ainda a advertência de King ${ }^{9}$ sobre as causas de erro que podem ser introduzidas em estudos econométricos pelo uso de deflatores inadequados em nosso meio.

Diante do exposto, resolvemos considerar apenas o valor corrente dos ganhos futuros perdidos.

Além de termos efetuado os cálculos do prejuízo econômico total das mortes verificadas em Salvador em 1970, resolvemos, para uma apreciação mais crítica e mais rigorosa do problema, levar em conta o fato de que nem todas aquelas mortes consideradas como "prematuras poderiam ter sido evitadas no nosso atual estágio de desenvolvimento. Para tanto, cotejamos as taxas de mortalidade de Salvador com as das demais capitais de estados e escolhemos para comparação Porto Alegre, por se tratar de uma cidade de extensão e população semelhantes a Salvador e que, a julgar pelos seus índices de mortalidade geral, de mortalidade infantil e de mortalidade por doenças infecciosas e transmissíveis, apresenta um nível de saúde bastante superior ao de Salvador. Porto Alegre pode ser portanto considerada, como um termo de comparação válido, um exemplo do melhor nível de saúde que uma cidade brasileira de cerca de um milhão de habitantes poderia, seguramente, ter atingido em 1970 (Tabela 2).

0 método que empregamos para esta comparação foi o de aplicar as taxas específicas de mortalidade para as várias causas em Porto Alegre em 1970, à população de Salvador, calculando, assim, o número de mortes esperadas, se Salvador tivesse alcançado em 1970 o mesmo nível de saúde de Porto Alegre. A seguir calculamos a diferença entre os óbitos esperados nas várias faixas etárias, pelas diferentes causas, com os óbitos efetivamente observados (Tabela 3), obtendo desta forma 0 número de mortes prematuras que poderiam ter sido evitadas em Salvador. mediante a aplicação de medidas profiláticas ou terapêuticas adequadas ou pela melhoria do nível de vida da população. A esta diferença, que representaria portanto a perda evitável de anos de vida produtiva (Tabela 4), aplicamos o mesmo tipo de cálculo anteriormente descrito para a estimativa do prejuízo econômico e que dá uma idéia mais justa e mais realista das perdas decorrentes das mortes evitáveis em Salvador no ano de 1970.

Procuramos ainda estudar os prejuízos econômicos decorrentes da mortalidade por causas específicas. Desta forma seria possível identificar os grupos de doenças que causando um maior prejuízo econômico mereceriam ser combatidas com prioridade pelas autoridades sanitárias. $\mathrm{Na}$ interpretação destes estudos, todavia. deverá ser necessariamente levado em conta o fato de que nem todas as causas de morte são igualmente suscetíveis de prevenção e/ou tratamento. Na realidade, a comparação entre as taxas de mortalidade por causas específicas em Salvador e em Porto Alegre demonstra que enquanto umas causas de óbito diminuem outras passam a assumir maior importância, sendo essas, em sua maioria, aquelas enfermidades para as quais a medicina ainda não encontrou métodos eficazes de prevenção ou de cura ${ }^{1 !}$. 
DUARTE DE ARAÚJO, J. - O custo da doença: repercussão económica no município de Salvador, BA, Brasil. Rev. Saúde pübl., S. Paulo, 9:155-68, 1975.

\section{TABELA 2}

Número de óbitos e coeficientes de mortalidade por grupos de causas nos municipios de Salvador, BA e Porto Alegre, RS - 1970

\begin{tabular}{|c|c|c|c|c|c|}
\hline & \multirow{2}{*}{$\begin{array}{c}\text { Grupos de causas e código da } \\
\text { Classificaça Internacional de } \\
\text { Doencas }\end{array}$} & \multicolumn{2}{|c|}{ Salvador } & \multicolumn{2}{|c|}{ Porto Alegre } \\
\hline & & $\begin{array}{l}\text { N.O de } \\
\text { obitos }\end{array}$ & $\begin{array}{l}\text { Taxa por } \\
100.000 \text { hab. }\end{array}$ & $\begin{array}{l}\text { N.o de } \\
\text { óbitos }\end{array}$ & $\begin{array}{c}\text { Taxa por } \\
100.000 \text { hab. }\end{array}$ \\
\hline 1. & $\begin{array}{l}\text { Doenças infecciosas e parasitá- } \\
\text { rlas }(000-136)\end{array}$ & 2.398 & 233,5 & 755 & 83.6 \\
\hline 2. & Neoplasias $(140-239)$ & 764 & 74,4 & 1.023 & 113,3 \\
\hline 3. & Diabetes mellitus $(250)$ & 127 & 12.4 & 1.34 & 14,8 \\
\hline 4. & $\begin{array}{l}\text { Avitaminoses e outras deficiên- } \\
\text { cias nutricionais }(260-269) \text {. Ane- } \\
\text { mias (280-285) }\end{array}$ & 236 & 23,0 & 176 & 19.5 \\
\hline 5 & $\begin{array}{l}\text { Doenças do sistema nervoso } \\
(320-389)\end{array}$ & 134 & 13,0 & 30 & 3,3 \\
\hline 6. & $\begin{array}{l}\text { Doencas do aparelho circulató- } \\
\text { rio }(300-458)\end{array}$ & 2.067 & 201,2 & 2.419 & 267,8 \\
\hline 7 . & $\begin{array}{l}\text { Doencas do aparelho respirató- } \\
\text { rio }(460-519)\end{array}$ & 739 & 71,9 & 596 & 66,0 \\
\hline 8 & $\begin{array}{l}\text { Doencas do aparelho digestivo } \\
(520-577)\end{array}$ & 222 & 21,6 & 160 & 17.7 \\
\hline 9. & $\begin{array}{l}\text { Doenças do aparelho gênito-uri- } \\
\text { nário }(580-629)\end{array}$ & 244 & 23.8 & 19 & 2,1 \\
\hline 10. & $\begin{array}{l}\text { Aborto e complicações da gravi- } \\
\text { dez e do parto }(630-678)\end{array}$ & 48 & 4,7 & 17 & 1,9 \\
\hline 11. & Anomallas congênitas (740-759) & 145 & 14,1 & 95 & 10,5 \\
\hline 12. & $\begin{array}{l}\text { Causas de mortalidade perinatal } \\
(760-779)\end{array}$ & 815 & 79,3 & 324 & 35,9 \\
\hline 13. & $\begin{array}{l}\text { Sintomas e afecçōes mal defini- } \\
\text { das }(780-796)\end{array}$ & 174 & 16.9 & 223 & 24,7 \\
\hline 14. & $\begin{array}{l}\text { Acidentes e outras mortes vio- } \\
\text { lentas (E 800-E 900) }\end{array}$ & 801 & 78,0 & 484 & 53,6 \\
\hline 15. & $\begin{array}{l}\text { Todas as outras causas (Resi- } \\
\text { duo) }\end{array}$ & 750 & 73,0 & 578 & 64,0 \\
\hline Tota & & 9.664 & 940.0 & 7.033 & 778,0 \\
\hline
\end{tabular}


DUARTE DE ARAÚJO, J. - O custo da doença: repercussão económica no municipio de Salvador, BA, Brasil. Rev. Saúde pübl., S. Paulo, 9:155-68, 1975.

TA BELA 3

Diferenca entre o numero de óbitos observado e o esperado para o município de Salvador. BA, aplicando as taxas de mortalidade de Porto Alegre. RS - 197ก

\begin{tabular}{c|c|c|c}
\hline $\begin{array}{c}\text { Grupos de causas e código da Classificacāo } \\
\text { Internacional de doenças }\end{array}$ & $\begin{array}{c}\text { obitos } \\
\text { observados }\end{array}$ & $\begin{array}{c}\text { obitos } \\
\text { esperados }\end{array}$ & $\begin{array}{c}\text { Diferença: } \\
\text { OBS-ESF }\end{array}$ \\
\hline
\end{tabular}

1. Doenças infecciosas e parasitárias $(000-136)$

2.398

764

127

3. Diabetes mellitus (250)

4. Avitaminoses e outras deficiências nutricionais (260-269). Anemias (280-285)

5. Doenças do sistema nervoso (320-389)

6. Doenças do aparelho circulatório (390-458)

7. Doenças do aparelho respiratório (460-519)

739

222

244 (580-629)

10. Aborto e complicacôes da gravidez e do parto $(630-678)$

11. Anomalias congênitas (740-759)

12. Causas de mortalidade perinatal (760-779)

13. Sintomas e afeccões mal definidas (780-796) (E 800 e $\mathrm{E} 999$ )

(Residuo)
48

145

815
858

1. 540

$1.163 \quad-399$

152

$-25$

200

36

33

101

2.750

$-683$

678

61

41

21

223

8

19

29

38

447

368

253

$-79$

551

250

657

93

Total 
DUARTE DE ARAƠJO, J. - o custo da doença: repercussão económica no municíplo de Salvador, BA, Brasil. Rev. Saúde puibl., S. Paulo, 9:155-68, 1975.

TABELA 4

Perda de anos de vida produtiva atribuida ao excesso de mortalidade em Salvador, BA. em comparação com Porto Alegre, RS, em 1970

\begin{tabular}{c|c|c|c}
\hline $\begin{array}{c}\text { Grupos etários } \\
\text { (anos) }\end{array}$ & Esperanca de vida & $\begin{array}{c}\text { Diferenca } \\
\text { de } \\
\text { óbitos }\end{array}$ & $\begin{array}{c}\text { Perda de anos de } \\
\text { vida produtiva }\end{array}$ \\
\hline
\end{tabular}
Sexo masculino

\begin{tabular}{|c|c|c|c|c|}
\hline Menos de 1 & 55,5 & 41.5 & 697 & $28.925,5$ \\
\hline $1-4$ & 60,8 & 49,3 & +144 & $7.099,2$ \\
\hline $5-14$ & 56,6 & $\mathbf{5 0 , 5}$ & $+\quad 62$ & $3.131,0$ \\
\hline $15-24$ & 51,7 & 44,5 & + & $2.714,5$ \\
\hline $25-34$ & 41,7 & $\mathbf{3 4 , 5}$ & 74 & $2.553,0$ \\
\hline $35-44$ & 33,3 & 24,5 & 62 & $1.519,0$ \\
\hline $45-54$ & 24,9 & 14,5 & 31 & 449.5 \\
\hline $55-64$ & 16,6 & 4,5 & 36 & 162,0 \\
\hline $65-74$ & 11,7 & - & 48 & - \\
\hline 75 e + & 4,2 & 一 & 50 & - \\
\hline Ignorado & - & - & 13 & - \\
\hline Total & - & - & +1.010 & $46.229,7$ \\
\hline
\end{tabular}

Sexo feminino

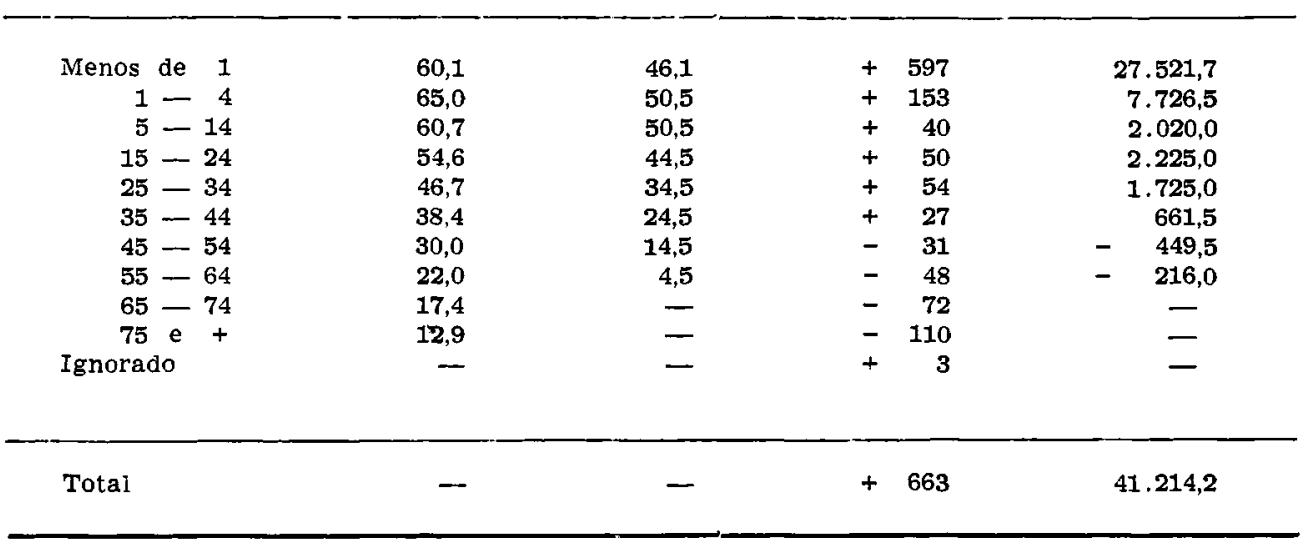

\section{RESULTADOS}

A estimativa dos anos de vida produtiva perdidos em decorrênçia da mortalidade precoce no Município de Salvador em 1970, levando em conta a idade do óbito e a esperança de vida para os vários grupos etários (Tahela 1) foi de
135.167,1 anos para o sexo masculino e de $116.336,1$ para o sexo feminino. Antes de calcular o valor monetário desta perda foi feita a correção para levar em conta o problema do desemprego.

No caso das mulheres engajadas em atividades domésticas, no próprio lar, atribuímos ao seu trabalho o mesmo valor 
DLARTE DE ARACJO, J. - o custo da doença: repercussão econômica no município de Salvador, BA, Brasil. Rev. Saúde públ., s. Paulo, 9:155-68, 1975.

do daquelas que exerciam atividade remunerada. Desta forma obtivemos o prejuizo econômico total decorrente da mortalidade precoce, expresso em perda dos ganhos futuros, em seu valor atual (cruzeiros de 1970), sem aplicar taxas de desconto, a qual foi a seguinte:

- Sexo masculino - Cr\$319.527.382,45

- Sexo feminino (inclusive as atividades domésticas) - Cr\$ 169.308.722.41

- Sexo feminino lexclusive atividades domésticas) - Cr\$ 71.254.339.54

- Ambos os sexos (inclusive atividade doméstica feminina) -

Cr\$ $\$ 88.880 .335 .84$

- Ambos os sexost exclusive atividade doméstica feminina) -

Cr\$391.781.721.99

Como já tivemos oportunidade de mencionar, este cálculo tomou como ponto de partida a premissa de que todos os indiríduos que morreram antes de atingir a esperança média de vida para o seu grupo etário, constituiram uma perda de anos de trabalho correspondente à diferença entre a idade do óbito e a respectiva esperança de vida. descontados os anos abaixo de 14 e acima de 65 .

Pareceu-nos, todaria, que a hipótese de que todos os indivíduos deveriam atingir o limite de vida média ia de encontro ao próprio conceito de vida média ou de esperança média de vida em determinado grupo etário. Ademais. a vida média estimada para Salvador, dadas as limitaçóes inerentes aos cálculos deste indicador quando as estatísticas de mortalidade não são completas e fidedignas, talvez não fosse a expressão real de nossa situação sanitária e representasse, antes, um ideal ainda não atingido ${ }^{16}$.

Diante dessas possíveis restrições. e dos argumentos apresentados na descrição da metodologia. é que partimos para outro cálculo, de natureza comparativa. entre a mortalidade de Salvador e a de Porto Alegre, aplicando o método anteriormente descrito.

Este cálculo mostrou, em 1970, um excesso de 1973 óbitos em Salvador. Em outras palavras verificaram-se em Salvador 9.664 óbitos quando seriam esperados apenas 7.991. caso se aplicassem à sua população, as taxas de mortalidade para cada grupo de doenças verificadas em Porto Alegre (Tabela 3).

0 estudo da distribuição deste excesso de óbitos pelos grupos etários, levando em conta a ocorrência das diversas causas de óbitos nas diferentes idades, permitiu estimar a perda de anos de vida produtiva verificada em Salvador, como resultado dos seus coeficientes específicos de mortalidade, mais elevados que o de outra cidade brasileira demograficamente comparável. Esta perda foi de $46.229,7$ anos para o sexo masculino e de $41.214,2$ para o sexo feminino (Tabela 4). O cálculo do prejuízo econômico. para ambos os sexos. foi feito utilizando a mesma metodologia anteriormente descrita.

Os resultados da estimativa da perda econômica decorrente da diferença em níreis de mortalidade entre Salvador e Porto Alegre foram os seguintes:

- Sexo masçulino - Cr\$ 109.284.381.61

- Sexo feminino (inclusive atividades domésticas ) - Cr\$ 59.980.712.58

- Sexo feminino (exclusive atividades domésticas) - Cr\$ 25.243.147.14

- Ambos os sexos (inclusive atividade doméstica feminina) - ....... Crs 169.265.094,19

- Ambos os sexos (exclusive atividade doméstica feminina) - $\ldots . .$. . Cr\$ $134.527 .528,75$

O estudo comparativo dos efeitos das taxas de mortalidade de Salvador e de 
DUARTE DE ARACJO, J. - O custo da doenca: repercussão económica no município de Salvador, BA, Brasil. Rev. Saúde píbl., S. Paulo, 9:155-68, 1975.

Porto Alegre sobre a perda de anos de vida produtiva, e de ganhos futuros, permitiu também a observação de quais as causas de morte que mais contribuiram para o prejuízo econômico.

Foram selecionadas para este estudo as três causas de óbitos que de acordo com os dados da Tabela 3 contribuiram com a maior parcela da diferença total: as doenças infecciosas e parasitárias, as causas de mortalidade perinatal e as mortes por acidentes e outras violências. Essas três causas em conjunto levaram a uma perda de vida produtiva de $44.866,3$ anos no sexo masculino e' de 38.604 .3 no sexo feminino (Tahela 5).

As estimativas feitas a partir dos dados expressos na Tabela 5, evidenciam a magnitude dos prejuízos resultantes dessas três causas que. conjuntamente, atingem Cr\$163.799.208.88 se incluirmos o valor do trabalho doméstico e Cr\$ .... 129.785.026.52 em caso contrário.

\section{DISCLSSAO E COMENTARIOS}

A estimativa dos prejuízos econômicos associados com a mortalidade precoce no município de Salvador, em 1970, dá bem uma medida da magnitude de um problema que até há bem pouco havia merecido escassa atenção na literatura médica brasileira.

O prejuízo total decorrente dos óbitos verificados antes da expectativa média de vida nos diversos grupos etários, para ambos os sexos, considerando o valor do trabalho das donas de casa como idêntico ao das mulheres empregadas e assalariadas. atingiu a elevada cifra de $\mathrm{Cr} \$$ 488.880.335.84. A alternativa de não atribuir valor econômico ao trabalho doméstico reduz esta cifra para $\mathrm{Cr} \$ \ldots$. 391.781.721.99. em moeda de 1970. Como termo de comparação, estes valores correspondem respectivamente a $65.77 \%$ e a $52.70 \%$ da receita do Estado da Bahia em 1970. e a $6.63 \%$ e $5.31 \%$ do
Produto Interno Bruto estimado para o Estado naquele ano. Correspondem ainda os mesmas valores a 13.87 vezes e 11.12 vezes, respectivamente, as despesas estaduais com o setor saúde em 1970. na Bahia. Merece atenção especial o fato de que $46,23 \%$ da perda de anos de vida de trabalho produtivo no sexo masculino. e $49.71 \%$ no sexo feminino. verificam-se no grupo etário de menos de $I$ ano.

As estimativas feitas com base na comparação entre as taxas de mortalidade de Salvador e as de Porto Alegre, no mesmo ano, objetivando obter uma visão mais realista do custo da mortalidade. e considerando apenas os óbitos que poderiam ter sido evitados no atual estágio de desenvolvimento do nosso país. revelaram, ainda, um considerável excesso de mortalidade cujo valor econômico foi calculado em Cr\$ 169.265.094,19, caso seja incluido o valor das atividades domésticas e em Cr\$134.527.528,75. excluindo o valor econômico das atividades das donas de casa. Essas cifras. embora bem mais reduzidas, representam ainda $22,77 \%$ e $18.09 \%$, respectivamente da receita estadual em 1970. e $2,29 \%$ e $1.82 \%$ do Produto Interno Bruto estimado para o Estado naquele ano. Ademais. correspondem elas respectivamente a 4.8 e 3,8 vezes os gatos estaduais com saúde em 1970.

A comparação com Porto Alegre permitiu ainda uma apreciação da importância econômica da mortalidade por grupos de causas, destacando-se as doenças infecciosas e parasitárias, as causas de mortalidade perinatal, e os acidentes e mortes riolentas. como as principais causas do excesso de mortalidade em Salvador. O conjunto destas três principais causas representou um prejuízo de Cr\$ ........ 163.799.208.88. incluindo o trabalho doméstico e de Cr\$ 129.785.026,52, sem incluir o trabalho doméstico correspondendo respectivamente a $22.03 \%$ e $17.46 \%$ da receita estadual em 1970. As doenças infecciosas e parasitárias por si só foram 


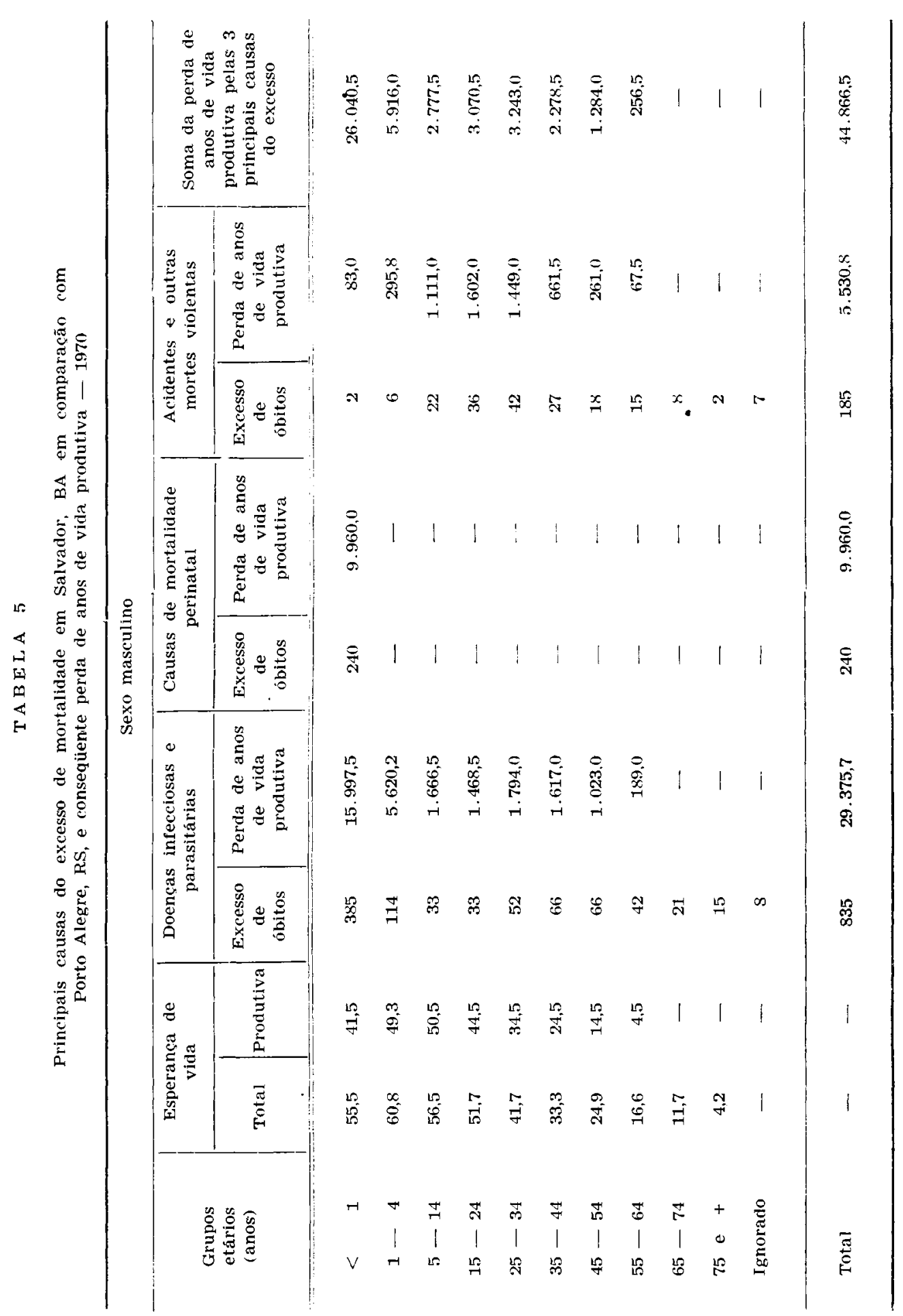


DUARTE DE ARAÚJO, J. - o custo da doença: repercussão econômica no município de Salvador, BA, Brasil. Rev. Saúde públ., S. Paulo, 9:155-68, 1975.

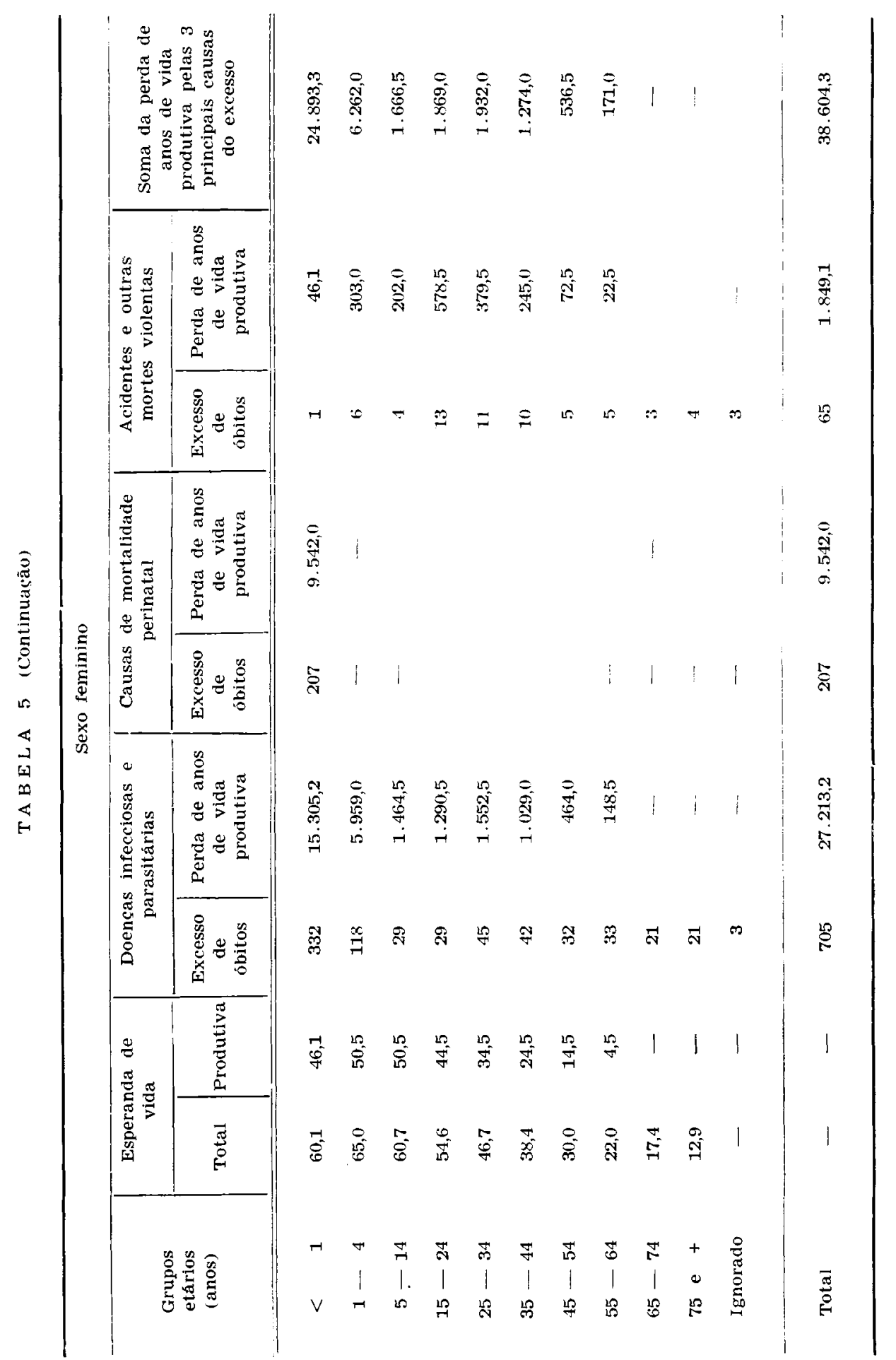


DUARTE DE ARAÚJo, J. - O custo da doença: repercussão econômica no municipio de Salvador, BA, Brasil. Rev. Saúde públ., S. Paulo, 9:155-68, 1975.

responsáveis por uma perda de $\mathrm{Cr} \$ \ldots$.... $110.599 .933,08$ ou $\mathrm{Cr} \$ 88.188 .798,45 \mathrm{con}-$ forme se inclua ou não o valor do trabalho doméstico. Esses últimos resultados confirmaram também a observação de que as repercussões econômicas da mortalidade são maiores nos grupos etários mais jovens. Mais ainda, eles demonstraram que o maior prejuízo resultante da mortalidade deve-se às doenças transmissíveis, as quais podem ser erradicadas ou controladas mediante a adoção dos meios apropriados ${ }^{1}$. Esses achados, utilizando metodologia assaz simples e de fácil aplicação, oferecem um subsídio valioso ao processo de determinação das prioridades na aplicação de recursos no setor saúde.

O estudo comparativo com Porto Alegre mostrou ainda que à medida que as providências acima apontadas surtem os seus efeitos, passam a surgir outros problemas de saúde como causa de mortalidade, notadamente as doenças do aparelho circulatório e as neoplasias malígnas, as quais passarão a merecer maior atenção das autoridades sanitárias à proporção em que cresça a sua importância como fator de morbidade e de prejuízo para a economia.

Convém relembrar que os achados que ora comentamos referem-se apenas ao Município de Salvador, correspondendo tão só a $13,54 \%$ da população do Estado, e onde se verificaram apenas 9.664 óbitos. A falta de dados fidedignos de estatística vital para os municípios do jnterior torna inviável a realização de estimativas seguras para todo o Estado. Admitindo-se. porém. para efeito apenas de discussão. que as taxas de mortalidade no interior do Estado. onde vivem os $86,46 \%$ restantes da população. sejam iguais à da Capital. uma hipólese otimista, teríamos que calcular o prejuízo decorrente de 61.629 óbitos e isto representaria uma perda economica 6,3 vezes maior do que a que calculamos com os dados da Capital. Estimativa semelhante. com resultados ainda mais dramáticos. poderia ser feita para a população do país como um todo. Todavia, estudos desta natureza devem, sempre que possível, evitar os cálculos dessa ordem e valer-se apenas de dados concretos, de validez indiscutível.

Fica assim patente que, diante da possibilidade de que as repercussões da mortalidade precoce constituam uma considerável perda econômica, torna-se recomendável a aplicação de metodologia semelhante à usada nesta tese, em âmbito regional ou mesmo nacional. 0 objetivo desses estudos seria melhor estimar a magnitude do problema, melhor conhecer as suas principais causas e assim poder planejar de modo mais racional a aplicação dos recursos financeiros da União, do Estado ou dos Municípios, para resolver os problemas prioritários de cada área. Vale no particular assinalar o considerável e exemplar esforço que vem sendo desenvolvido pelo Centro Regional de Estatística de Saúde do Nordeste (CRESNE) para dotar os administradores e planejadores de saúde da região de estatísticas fidedignas, representativas de toda sua área. as quais irão permitir em futuro próximo a quantificação, não só das perdas decorrentes do fenômeno doença/morte. como também dos benefícios decorrentes da aplicação de recursos financeiros em programas de saúde no Nordeste.

Convém destacar que, por dificuldade de ordem tanto conceitual como metodológica, os nossos dados não incluem a perda econômica do investimento feito na formação dos indivíduios que morrem antes de entrar na força de trahalho. Estes custos incluem alimentação, habitação. educação e os cuidados médicos, bem como a atenção pessoal dos pais, de valor econômico inestimável. Esse custo, que foi particularmente valorizado por Sauvy ${ }^{1 i}$ e por Hanlon ${ }^{7}$, representa um vultoso investimento, que se perde pela morte verificada antes da idade produtiva e que. numa avaliação completa do custo da mortalidade, deveria ser somado às perdas decorrentes dos ganhos futuros não reali- 
DLARTE DE ARAÚJO, J. - O custo da doença: repercussão econômica no municipio de salvador. BA, Brasil. Rev. Saúde públ., S. Paulo, 9:155-68, 1975.

zados. Hanlon ${ }^{7}$, utilizando custos de 1955, estimou para os Estados Linidos, em LS\$ 60,000.00 o investimento médio feito em cada indivíduo até atingir a idade de 18 anos. Infelizmente, não dispomos de dados empíricos que nos permitam tentar sequer uma estimativa aproximada do valor equivalente para a população brasileira. Todavia, não deixamos de concordar em que idealmente uma avaliação rigorosa do custo total da mortalidade deva, também, incluir a parcela a que acabamos de aludir.

A não inclusão de tal parcela é uma demonstração de que os cálculos aqui apresentados, longe de representarem um exagero na avaliação dos custos da mortalidade, constituem apenas uma estimativa mínima daqueles prejuízos que, se apurados com maior rigor, evidenciarão uma sangria ainda mais séria nos recursos eco- nômicos do país. estado ou região estudados.

Para os objetivos a que nos propusemos. todavia, as estimativas mínimas apresentadas são suficientes, pois demonstram o considerável ônus que a mortalidade por doenças perfeitamente evitáveis, representa para um Estado como a Bahia que se encontra empenhado em notável esforço para a superação do subdesenvolvimento. Tossos achados demonstram que medidas rugentes se fazem necessárias para incluir as atividades de saúde pública como parte integrante deste esforço. $\mathrm{Na}$ atual conjuntura do nosso estado, e acreditamos poder estender esta afirmativa a todo o país, existe forte evidencia de que os gastos no setor saúde, dentro de uma escala racional de prioridades, devem ser vistos pelos planejadores como investimento e não como despesa de consumo.

\section{RSPU-B/256}

DLARTE DE ARALiJo, J. - [The cost of disease: economical consequences in the city of Salvador, BA, Brazll]. Rev. Saúde públ., S. Paulo, 9:155-68, 1975

SUMMARY: The economical consequences of mortality in the city of Salvador, Brazil, in 1970 were studied. A comparison was drawn between the cities of Salvador and Porto Alegre and the cost of the excess in Salvador was evaluated. The results showed that the economical losses associated with mortality from preventable diseases were important and lead to the conclusion that health service budgets should be considered as investments and not consumption Expenditures.

UNITERms: Cost and cost analysis. Economics. medical. Salvador. BA (Brazll).

\section{REFERENCIAS BIBLIOGRAFICAS}

1 BENENSON, A.S., ed. Control of communcable diseases in man. $7^{\text {th }}$ ed Washington, D.C. The American Public Health Association, 1970,

2 DUARTE DE ARAUJO, J. - O custo da doença: revisão de literatura. Rev. Saude públ., S. Paulo, 9: 1975

3 FEIN, R - Economics of mental llness New York. Basic Books Inc $195 x$
4. FUNDAC̣̃O IBGE - Anuário Estatisti(o) do Brasil, 1971. Rio de Janeiro. 1972 .

5. FUNDACAO IBGE - Pesquisa Nacional por Amostra de Domicilios Regnio $V$ Rin de Janeiro, 1970 (Documento do GEPD n. 47 )

6 GRIFFITH. D.H.S et al. - Contribution of hedith to development. Int. $J$ Hith serv. 1:253-70, 1971. 
DUARTE DE ARAúJO, J. - O custo da doença: repercussão económica no município de salvador, BA, Brasil. Rev. Saúde pribl., S. Paulo, 9:155-68, 1975.

7. HANLON, J.J. - Principles of public health administration, $5^{\text {th }}$ ed., St. Louis. The C. V. Mosby Co, 1969. p. 112-27.

8. JOHNSON, T. - Returns from investment in human capital. Amer. Econ. Rev., 60:546-60, 1970 .

9. KING, K. - O emprego de deflatores inadequados, o problema de erro comum nas variáveis em estudos econométricos. Pesquisa e Planejamento, 1:403-14, 1971 .

10. KLARMAN, H.E. - Syphilis control programs. In: DORFMAN, R., ed. - Measuring Eenefits of Government Investments. Washington, D. C., The Brookings Institution, 1965. p. $367-414$.

11. LANGONI, C.G. - Como o Brasil pode crescer a $9 \%$ ao ano poupando apenas $15 \%$ do produto. Estudos EConômicos Brasileiros Seleções APEC, $8: 27-31,1971$.

12. MILLER, H.P. - Lifetime income and economic growth. Amer, Econ. Rev., $55: 835-44,1965$.
13. PAGLIN, M. - Surplus agricultural labor and development: fact and theories. Amer. Econ. Rev.: 35:814-34, 1965.

14. PUFFER, R.R. \& GRIFFITH, G.W. Patterns of urban mortality: Report of the inter-american investigation of mortality. Washington, Pan American Health organization, 1967. (Scient. publ., 151).

15. RICE, D.P. - Estimating the cost of illness. Washington, D.C., U.S. Government Priting Office, 1966. (Public Health Service Publ., 947-56)

16. SANTOS, J.L.F. \& SINGER - A dinamica populacional de Salvador: Programa de Recursos Humanos da Universidade Federal da Bahı́, São Paulo, CEDIP-CEBRAP, 1970.

17. SAUYY, A. - Thérie Génerale de la population: Economie et population. Paris. Presses Universitaires de France, 1952. v. 1.

Recebillo para publicaşăo em 20-12-197! Aprovado para publicaç̃o em 13-01-1975 